Research Article

\title{
Study on PPG Biometric Recognition Based on Multifeature Extraction and Naive Bayes Classifier
}

\author{
Junfeng Yang $\mathbb{D},{ }^{1}$ Yuwen Huang, ${ }^{1}$ Ruili Zhang, ${ }^{1}$ Fuxian Huang, ${ }^{1}$ Qinggang Meng $\mathbb{D}{ }^{2}$ \\ and Shixin Feng ${ }^{1}$
}

${ }^{1}$ School of Computer, Heze University, Heze 274015, China

${ }^{2}$ School of Software, Shandong University, Jinan 250101, China

Correspondence should be addressed to Qinggang Meng; 201984000191@sdu.edu.cn

Received 5 January 2021; Revised 23 March 2021; Accepted 22 April 2021; Published 6 May 2021

Academic Editor: Antonio J. Peña

Copyright (C) 2021 Junfeng Yang et al. This is an open access article distributed under the Creative Commons Attribution License, which permits unrestricted use, distribution, and reproduction in any medium, provided the original work is properly cited.

\begin{abstract}
Nowadays, the method of simple-feature extraction has been extensively studied and is used in PPG biometric recognition; some promising results have been reported. However, some useful information is often lost in the process of PPG signal denoising; the time-domain, frequency-domain, or wavelet feature extracted is often partial, which cannot fully express the raw PPG signal; and it is also difficult to choose the appropriate matching method. Therefore, to make up for these shortcomings, a method of PPG biometric recognition based on multifeature extraction and naive Bayes classifier is proposed. First, in the preprocessing of the raw PPG data, the sliding window method is used to rerepresent the raw data. Second, the feature-extraction methods based on time-domain, frequency-domain, and wavelet are analysed in detail, then these methods are used to extract the time-domain, frequency-domain, and wavelet features, and the features are concatenated into a multifeature. Finally, the multifeature is normalized and combined with classifiers and Euclidean distance for matching and decision-making. Extensive experiments are conducted on three PPG datasets, it is found that the proposed method can achieve a recognition rate of $98.65 \%, 97.76 \%$, and $99.69 \%$ on the respective sets, and the results demonstrate that the proposed method is not inferior to several state-of-the-art methods.
\end{abstract}

\section{Introduction}

Since biometrics system based on human physiological or behavioural characteristics is more reliable and safer than traditional identification technology, certain distinctive features of our body or behavioural attributes, such as fingerprint [1], face [2], voice [3], iris [4], lip print [5], gait motion [6], palm vein [7], finger vein [8], electroencephalography (EEG) [9], and electrocardiography (ECG) $[10,11]$, are viewed as means of human identification. These applications based on biometric approaches provide a promising and convincing future of human recognition. However, a fingerprint can be modified with latex, face recognition can be faked with an artificially disguised, voice can be imitated, and the EEG and ECG-based methods are to some extent complicated to acquire the biosignals. Therefore, photoplethysmography (PPG) biometric recognition has received considerable attention in authentication for personal privacy and fraud prevention.
PPG is a noninvasive electrooptical methodology which provides much physiological information about the human body. In recent years, PPGs were proven to have competency to distinguish individuals [12]. As a new biometrics technology, PPG has been verified by studies for its universality, uniqueness, robustness, and adaptability $[13,14]$. Compared to the ECG, EEG, and so on, the PPG signal can be acquired at a low cost and is more accessible and more portable device. In addition, the PPG signal can be collected from different positions in the human body such as fingertips, wrist, or earlobes. And PPG measurements only need to be acquired from one side of the body, allowing it to be used in a larger number of human recognition scenarios. Therefore, PPG signal has more practical application and appealing $[15,16]$.

To the best of our knowledge, Gu et al. [1] were the first to study PPG for human verification, considering four feature parameters and achieving 94\% accuracy. Since then, 
many scholars have researched PPG biometric recognition. For example, some scholars took low pass filter (LPF), Butterworth filter (BWF), the first derivative (FD), the second derivative (SD), finite infinite response (FIR) filter, peak detection (PD), and sliding window to signal preprocessing; some scholars took time-domain feature (TDF) analysis, frequency-domain feature (FDF) analysis, discrete wavelet transform (DWT), continuous wavelet transform (CWT), linear discriminant analysis (LDA), Karhunen-Loève transform (KLT), discrete cosine transform (DCT), statistical curve width (SCW), and three-layer features based on sparse softmax vector for feature extraction; some scholars took $\mathrm{k}$-nearest neighbor (k-NN), majority voting (MV), linear discriminating classifier (LDC), support vector machine (SVM), Bayes network (BN), Pearson's distance (PearsD), Manhattan distance, Euclidean distance, naive Bayes (NB), radial basis function (RBF), multilayer perceptron (MLP), decision tree (DT), random forest (RF), gradient boosted trees (GBT), isolation forest (IsFrst), and so on to classification. The technologies or methods in the related literature are summarized for preprocessing, feature extraction and classification in Table 1. In addition, some scholars proposed PPG biometric methods based on deep learning [16, 31-34] and achieved good recognition performance. However, deep learning is not easy to train on small-scale data, and it has too many hyperparameters that need intricate adjustment, which requires powerful computational resources. Therefore, this paper does not consider deep learning methods.

Table 1 shows that, in the current PPG biometrics researches, firstly, low pass and Butterworth filters are mainly used to reduce the PPG signal noise, or the first and SD are used to do the PPG signal preprocess, but these methods often cannot remove motion artifacts, baseline wanders, and power line interference noises at the same time and often cause the signal to lose useful information; secondly, the time-domain or the FDF analysis and wavelet transform are mainly used for feature extraction; however, the simple features extracted by these methods are often partial, which cannot fully express the raw PPG signal; thirdly, in terms of matching or classification, there are often many technologies or methods to be selected, but how to choose a technique or method that can achieve the best performance is also a difficult problem. Therefore, to make up for these shortcomings, a method of PPG biometrics based on multifeature extraction and naive Bayes classifier (NBC) is proposed herein. The contributions of this paper are summarized as follows.

First, we use the sliding window method to rerepresent the PPG raw data and analyse the influence of sliding window size and sliding step size on the recognition rate.

Second, we propose a novel multifeature-extraction method to efficiently capture time-domain, frequency-domain, and wavelet signal characteristics and combine them to form a multifeature.

Finally, extensive experiments are conducted on three PPG datasets; the results demonstrate that the proposed method is not inferior to several state-of-the-art methods.

The remainder of this paper is organized as follows. Section 2 describes the proposed method in detail. Section 3 reports the experimental results and provides a
TABLE 1: Summary of technologies or methods used for PPG biometrics.

\begin{tabular}{|c|c|c|}
\hline Stage & Technology or method & Literature \\
\hline \multirow{7}{*}{ Preprocessing } & LPF & [17-20] \\
\hline & BWF & {$[13,21,22]$} \\
\hline & $\mathrm{FD}$ and $\mathrm{SD}$ & {$[17,23]$} \\
\hline & LPF, FD, and SD & {$[24]$} \\
\hline & FIR, FD, and SD & {$[14,25,26]$} \\
\hline & $\mathrm{PD}$ & [27] \\
\hline & Sliding window & {$[28]$} \\
\hline \multirow{8}{*}{ Feature extraction } & TDF & $\begin{array}{c}{[14,19,23-25,} \\
27,29]\end{array}$ \\
\hline & TDF and FDF & {$[26]$} \\
\hline & DWT & {$[18]$} \\
\hline & CWT & {$[21]$} \\
\hline & LDA & {$[27]$} \\
\hline & TDF and KLT & {$[22]$} \\
\hline & SCW, FDF, and TDF & {$[20]$} \\
\hline & Sparse softmax vector & [28] \\
\hline \multirow{11}{*}{$\begin{array}{l}\text { Matching or } \\
\text { classification }\end{array}$} & $\mathrm{k}-\mathrm{NN}$ & {$[14,18,23]$} \\
\hline & $\mathrm{k}-\mathrm{NN}$ and $\mathrm{MV}$ & [27] \\
\hline & LDC & {$[25]$} \\
\hline & SVM & {$[18,26]$} \\
\hline & $\mathrm{BN}$ and $\mathrm{k}-\mathrm{NN}$ & {$[24]$} \\
\hline & PearsD & {$[21]$} \\
\hline & $\begin{array}{l}\text { Manhattan distance and } \\
\text { Euclidean distance }\end{array}$ & {$[22]$} \\
\hline & $\mathrm{BN}, \mathrm{NB}, \mathrm{RBF}$, and MLP & [19] \\
\hline & DCT & {$[30]$} \\
\hline & SVM, GBT, and IsFrst & {$[20]$} \\
\hline & $\mathrm{k}-\mathrm{NN}, \mathrm{NB}, \mathrm{RF}$, and LDC & [28] \\
\hline
\end{tabular}

comprehensive analysis. Finally, Section 4 presents the conclusions and future work.

\section{Proposed Methods}

To perform PPG biometric recognition, a PPG biometrics framework was designed. First (mentioned in Section 2.1), the raw PPG data are rerepresented by the sliding window method. Second (mentioned in Section 2.2), the multifeature is generated from the rerepresented PPG data. Finally (mentioned in Section 2.3), the matching and decision are performed after the multifeature normalized. The block diagram of the proposed PPG biometrics process is shown in Figure 1. In the following subsections, preprocessing of the raw PPG data, multifeature extraction and matching, and so on will be detailed.

\subsection{Preprocessing}

2.1.1. Rerepresenting Data Using Sliding Window Method. There are various sources of artifacts that interfere with PPG signal acquisition, including motion artifacts, baseline wander, and power line interference, so many scholars used various denoising methods to preprocess data. However, some useful information may be removed in the process of denoising. Therefore, to avoid the situation, this paper uses the sliding window [28] method to rerepresent the raw PPG data, and it is no longer necessary 


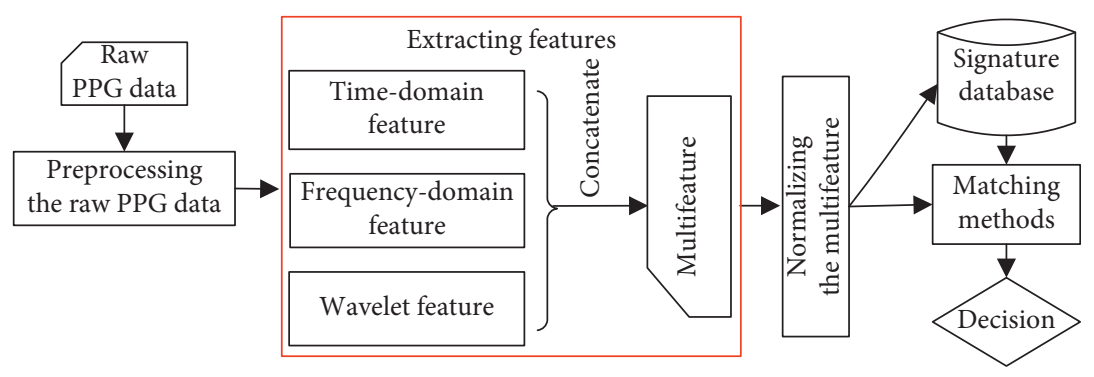

Figure 1: Block diagram of the proposed PPG biometrics process.

to denoise. Figure 2 shows the working process of the sliding window method, $W_{\text {size }}$ is the size of sliding window, and $S_{\text {step }}$ is the step size of sliding. After sliding window scanning, a large number of samples are produced from the raw PPG data of each subject, which can also make up for the lack of samples in the training process, and these samples constitute a matrix that is rerepresented.

2.1.2. Influence Factor. In the process of rerepresenting the raw PPG data using the sliding window method, the size of the sliding window and the sliding step size are the main influence factors of recognition rate. With the increase of the sliding window size, the recognition rate will also be improved but will be in a relatively stable state after the window size reaching a certain extent. With the increase of sliding step size, the recognition rate will decrease; when the sliding step size is smaller, the recognition effect is better. We analyse $W_{\text {size }}$ and $S_{\text {step }}$ in detail in Section 3.3.

2.2. Feature Extraction. To the best of our knowledge, timedomain, frequency-domain, and wavelet features are simultaneously extracted for the PPG biometrics, which has not yet been achieved. Therefore, this section studies multiple features' extraction and their concatenation.

2.2.1. TDF. TDF has a wide application in PPG biometrics researches $[14,19,23-25,27,29]$. It is simple in feature extraction, does not need any transformation, and is directly extracted from the preprocessed PPG signals. Table 2 lists the formulas for extracting $17 \mathrm{TDF}$ of a sample, let $x_{n}=\left[x_{n}^{0}\right.$, $\left.x_{n}^{1}, x_{n}^{2}, \ldots, x_{n}^{i}, \ldots, x_{n}^{N-1}\right]^{T}$ denote the $\mathrm{n}$-th subject, where $x_{n}^{i}$ is the amplitude corresponding to the $\mathrm{i}$-th sample of the $\mathrm{n}$-th subject, $\mathrm{N}$ is the total number of sample points after time-domain segmentation, $\max$ is the function of taking the maximum value, $\mathrm{min}$ is the function of taking the minimum value, and the relevant calculation formula is shown in Table 2. For convenience, the TDF extracted for the i-th sample of the $n$-th subject can be expressed as $\mathrm{TDF}_{n}^{i}=\left[F_{n}^{t 1}, F_{n}^{t 2}, F_{n}^{t 3}, \ldots, F_{n}^{t 17}\right]^{T}$. In general, the time-domain analysis is more intuitive, but the discriminability of TDF is weak.

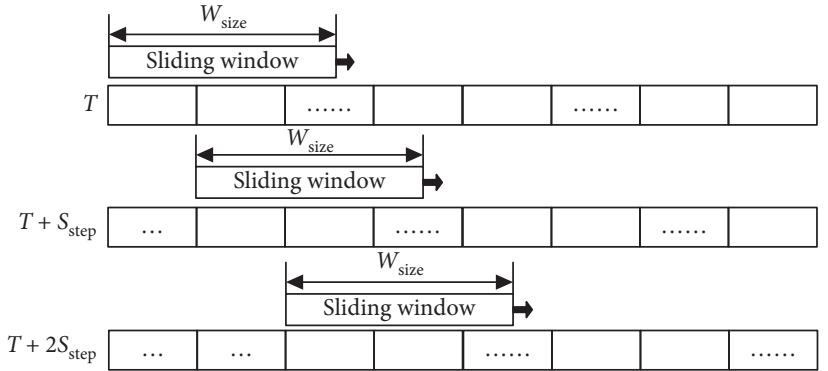

FIGURE 2: Working process of the sliding window.

2.2.2. FDF. The frequency-domain representation (spectrum) refers to breaking down a signal into its sinusoids. That is, the spectrum of a signal is a representation of its frequency content [35]. Four FDF parameters commonly used by researchers are gravity frequency, mean frequency, root mean square frequency, and frequency standard deviation. Therefore, in this paper, the spectrums of the PPG signal are first extracted by the fast algorithm of discrete Fourier transform (FFT), and then the four FDFs are extracted according to the formula given in Table 3. Let $x_{n}=$ $\left[x_{n}^{0}, x_{n}^{1}, x_{n}^{2}, \ldots, x_{n}^{i}, \ldots, x_{n}^{N-1}\right]^{T}$ denote the $\mathrm{n}$-th subject, where $x_{n}$ is time-domain segmentation and absolutely integrable, $x_{n}^{i}$ is the amplitude corresponding to the $\mathrm{i}$-th sample of the $\mathrm{n}$-th subject, $\mathrm{N}$ is the total number of the sample points of the segmentation, and $x_{n}^{i}$ s FFT can be expressed as follows:

$X_{n}^{i}=\left\{y \mid y=\sum_{j=1}^{N-1} x_{n}^{i}(j) e^{-\lambda(2 \pi k / N) i}, \quad k=0,1,2, \ldots, \mathrm{L}-1\right\}$,

where $L=N / 2$ and $X_{n}^{i}$ is a frequency amplitude of the $\mathrm{i}$-th sample of the $n$-th subject. The main disadvantage of FDF compared to TDF is the higher computational cost. For convenience, the FDF extracted for the i-th sample of the $\mathrm{n}$-th subject can be expressed as $\operatorname{FDF}_{n}^{i}=\left[F_{n}^{f 1}, F_{n}^{f 2}, F_{n}^{f 3}\right.$, $\left.F_{n}^{f 4}\right]^{T}$. It is thus clear that the frequency-domain representation is more concise, but the time characteristic of signal is lost after Fourier transform.

2.2.3. Wavelet Feature. Time-domain or frequency-domain characteristic cannot describe the time varying of signal and cannot be localized for signal analysis. That is, their time- 
TABLE 2: Calculation formula of the time-domain feature (TDF) of a sample.

\begin{tabular}{|c|c|c|}
\hline Label of the feature & Name of the feature & Formula \\
\hline$\overline{F_{n} t 1}$ & Max value & $F_{n}^{t 1}=\max \left(x_{n}^{i}\right)$ \\
\hline$F_{n}^{t 2}$ & Min value & $F_{n}^{t 2}=\min \left(x_{n}^{i}\right)$ \\
\hline$F_{n}^{t 3}$ & Peak value (PV) & $F_{n}^{t 3}=P V_{n}(i)=\max \left(\left|x_{n}^{i}\right|\right)$ \\
\hline$F_{n}^{t 4}$ & Peak to peak value & $F_{n}^{t 4}=\max \left(x_{n}^{i}\right)-\min \left(x_{n}^{i}\right)$ \\
\hline$F_{n}^{t 5}$ & Mean value & $F_{n}^{t 5}=\bar{X}_{n}(i)=\frac{1}{N} \sum_{j=0}^{N-1} x_{n}^{i}(j)$ \\
\hline$F_{n}^{t 6}$ & Square root amplitude (SRA) & $F_{n}^{t 6}=\operatorname{SRA}_{n}(i)=\left(\frac{1}{N} \sum_{j=0}^{N-1} \sqrt{\left|x_{n}^{i}(j)\right|}\right)^{2}$ \\
\hline$F_{n}^{t 7}$ & Mean amplitude (MA) & $F_{n}^{t 7}=\operatorname{MA}_{n}(i)=\frac{1}{N} \sum_{j=0}^{N-1}\left|x_{n}^{i}(j)\right|$ \\
\hline$F_{n}^{t 8}$ & Variance & $F_{n}^{t 8}=\frac{1}{N} \sum_{j=0}^{N-1}\left(x_{n}^{i}(j)-\bar{X}_{n}(i)\right)^{2}$ \\
\hline$F_{n}^{t 9}$ & Standard deviation (STD) & $F_{n}^{t 9}=\operatorname{STD}_{n}(i)=\sqrt{\frac{1}{N} \sum_{j=0}^{N-1}\left(x_{n}^{i}(j)-\bar{X}_{n}(i)\right)^{2}}$ \\
\hline$F_{n}^{t 10}$ & Root mean square (RMS) & $F_{n}^{t 10}=\operatorname{RMS}_{n}(i)=\sqrt{\frac{1}{N} \sum_{j=0}^{N-1}\left(x_{n}^{i}(j)\right)^{2}}$ \\
\hline$F_{n}^{t 11}$ & Skewness & $F_{n}^{t 11}=\frac{1}{N} \sum_{j=0}^{N-1}\left(\frac{x_{n}^{i}(j)-\bar{X}_{n}(i)}{\operatorname{STD}_{n}(i)}\right)^{3}$ \\
\hline$F_{n}^{t 12}$ & Kurtosis & $F_{n}^{t 12}=\frac{1}{N} \sum_{j=0}^{N-1}\left(\frac{x_{n}^{i}(j)-\bar{X}_{n}(i)}{\operatorname{STD}_{n}(i)}\right)^{4}$ \\
\hline$F_{n}^{t 13}$ & Waveform index & $F_{n}^{t 13}=\mathrm{RMS}_{n}(i) / \mathrm{MA}_{n}(i)$ \\
\hline$F_{n}^{t 14}$ & Pulse index & $F_{n}^{t 14}=P V_{n}(i) / M A_{n}(i)$ \\
\hline$F_{n}^{t 15}$ & Peak index & $F_{n}^{t 15}=\mathrm{PV}_{n}(i) / \mathrm{RMS}_{n}(i)$ \\
\hline$F_{n}^{t 16}$ & Margin index & $F_{n}^{t 16}=\mathrm{PV}_{n}(i) / \operatorname{SRA}_{n}(i)$ \\
\hline$F_{n}^{t 17}$ & Clearance index & $F_{n}^{t 17^{n}}=\mathrm{PV}_{n}(i) /\left(\operatorname{RMS}_{n}(i)\right)^{2}$ \\
\hline
\end{tabular}

TABle 3: Calculation formula of each FDF. $f_{n}^{i}$ is a frequency of the $\mathrm{i}$-th sample of the $\mathrm{n}$-th subject.

\begin{tabular}{lcc}
\hline Label of the feature & Name of the feature & Formula \\
\hline$F_{n}^{f 1}$ & Gravity frequency $(\mathrm{GF})$ & $F_{n}^{f 1}=\mathrm{GF}_{n}(i)=\left(\sum_{j=0}^{L-1}\left(f_{n}^{\mathrm{i}}(j) X_{n}^{i}(j)\right) / \sum_{j=0}^{L-1} X_{n}^{i}(j)\right)$ \\
$F_{n}^{f 2}$ & Mean frequency & $F_{n}^{f 2}=(1 / L) \sum_{j=0}^{L-1} X_{n}^{i}(j)$ \\
$F_{n}^{f 3}$ & RMS frequency & $F_{n}^{f 3}=\sqrt{\sum_{j=0}^{L-1}\left(\left(f_{n}^{i}(j)\right)^{2} X_{n}^{i}(j)\right) / \sum_{j=0}^{L-1} X_{n}^{i}(j)}$ \\
$F_{n}^{f 4}$ & Frequency standard deviation & $F_{n}^{f 4}=\sqrt{\sum_{j=0}^{L-1}\left(\left(f_{n}^{i}(j)-F G_{n}(i)\right)^{2} X_{n}^{i}(j)\right) / \sum_{j=0}^{L-1} X_{n}^{i}(j)}$ \\
\hline
\end{tabular}

frequency resolution is not higher. While wavelet functions are localized in the time-domain as well as in the frequencydomain [36], it should be noted that successive details are never reanalysed in the orthogonal wavelet decomposition procedure [37]. Therefore, this section discusses the wavelet packet which can be used for multiresolution decomposition and proposes a feature-extraction approach based on wavelet packet decomposition.

The wavelet packet decomposition is a generalization of wavelet analysis that offers a more precise analysis method for signals; wavelet packet atoms are waveforms indexed by frequency, scale, and position three parameters [37]. For a given orthogonal wavelet function, a set of wavelet packet bases can be generated, each of which offers a particular way of coding signals, preserving global energy, and reconstructing exact features; the wavelet packets can be used for numerous decompositions of a given signal. In the process of wavelet packet decomposition, one-dimensional frequency coefficients are usually split into two parts, one part is referred to as an approximate coefficient vector, and the other part is referred to as detail coefficient vectors which are obtained. And then the next step is to further split the approximation coefficient vector into two parts, and the detail coefficient vector is also decomposed into two parts using the same approach as in approximation vector splitting. In this way, they are decomposed level by level until the requirements are met. However, it should be pointed out that not all wavelet bases are suitable for wavelet decomposition, so it is necessary to select appropriate wavelet bases. Figure 3 shows the schematic diagram of wavelet packet decomposition of three levels for one-dimensional signals [37], $\mathrm{S}$ is the raw signal before decomposition, $\mathrm{A}$ is the high-frequency signal after decomposition, $D$ is the lowfrequency signal after decomposition, and the footnote is the number of levels of decomposition.

The wavelet feature including frequency-band energy ratio, energy entropy, scale entropy, and singular entropy is extracted as follows:

(1) The eight frequency subbands are obtained for each sample by three-level wavelet packet decomposition based on wavelet basis db8 (to this end, the mother wavelets such as sym8, db8, coif5, bior3.9, and dmey were studied, and experiments show that $\mathrm{db} 8$ is the best), and the wavelet packet decomposition coefficients are extracted from the subbands, which are reconstructed, and then they are denoted by $R W P D C_{n}^{\mathrm{i}}$ (reconstructed wavelet packet decomposition coefficient of the $\mathrm{i}$-th sample of the $\mathrm{n}$-th subject). 


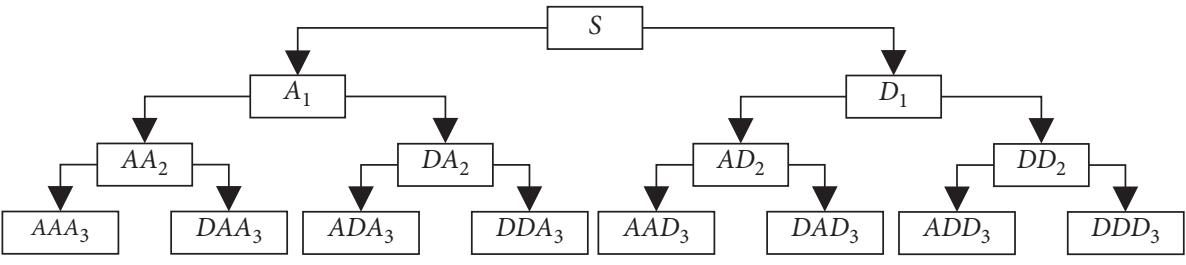

FIgURE 3: Wavelet packet decomposition tree at level 3.

(2) Energy ratios (ER) of the eight frequency subbands are calculated by the formula as follows:

$$
E R_{n}^{i}=\left\{y \mid=\frac{\left(R W P D C_{n}^{i}(j)_{2}\right)^{2}}{\sum_{k=1}^{8}\left(R W P D C_{n}^{i}(k)_{2}\right)^{2}}, \quad j=1,2,3, \ldots, 8\right\} .
$$

(3) Energy entropy (EE) is calculated by the formula as follows:

$$
\mathrm{EE}_{n}(i)=-\sum_{j=1}^{8} \mathrm{ER}_{n}^{i}(j) \times \log \mathrm{ER}_{n}^{i}(j)
$$

(4) Scale entropy (SE) of eight frequency subbands is obtained according to the wavelet packet decomposition coefficients without being reconstructed, which is denoted as $\mathrm{SE}_{n}^{i}=\left\{y \mid y=\operatorname{SE}_{n}^{i}(j), \quad j=1,2\right.$, $3, \ldots, 8\}$ ( $\mathrm{SE}$ is obtained directly through the wentropy function of MATLAB).

(5) Computing singular spectral entropy (SSE): firstly, the singular spectral vector (SSV) is obtained according to wavelet packet decomposition coefficient with reconstructed, which is denoted as $\operatorname{SSV}_{n}^{i}=\left\{y \mid y=\operatorname{SSV}_{n}^{i}\right.$ (j), $\quad j=1,2,3, \ldots, 8\}$, and then the singular spectral ratio (SSR) is calculated by the formula as follows:

$\operatorname{SSR}_{n}^{i}=\left\{y \mid y=\frac{\operatorname{SSV}_{n}^{i}(j)}{\sum_{j=1}^{8} \operatorname{SSV}_{n}^{i}(j)}, \quad j=1,2,3, \ldots, 8\right\}$.

Finally, the SSE is calculated by the formula as follows:

$$
\operatorname{SSE}_{n}(i)=-\sum_{j=1}^{8} \operatorname{SSR}_{n}^{i}(j) \times \log \operatorname{SSR}_{n}^{i}(j) .
$$

(6) The wavelet feature (WF) extracted for the i-th sample of the n-th subject can be expressed as $\mathrm{WF}_{n}^{i}=$ $\left[\mathrm{ER}_{n}^{1}, \mathrm{ER}_{n}^{2}, \ldots, \mathrm{ER}_{n}^{8}, \mathrm{EE}_{n}(i), \mathrm{SE}_{n}^{1}, \mathrm{SE}_{n}^{2}, \ldots, \mathrm{SE}_{n}^{8}, \mathrm{SSE}_{n}\right.$ (i) $]^{\text {t }}$.

2.2.4. Multifeature Concatenating. As previously mentioned, in the past researches, the methods of simple-feature extraction with time, frequency, or wavelet are often preferred, while the methods based on the simple-feature analysis have their own shortcomings. Therefore, in order to reduce the impact of the shortcomings, make the extracted features more approximately represent the raw PPG signal, and make PPG biometric recognition more accurate, after $\mathrm{TDF}_{n}^{i}, \mathrm{FDF}_{n}^{i}$, and $\mathrm{WF}_{n}^{i}$ are extracted separately, the next step is to concatenate them into one feature vector to form the multifeature (MF). For the i-th sample of the $\mathrm{n}$-th subject, the multifeature vector can be represented as $\mathrm{MF}_{n}^{i}=\left[\mathrm{TDF}_{n}^{i}\right.$; $\left.\mathrm{FDF}_{n}^{i} ; \mathrm{WF}_{n}^{i}\right]$.

2.2.5. Normalization. Due to the different dimensions of indexes, if the various feature extracted is directly sent to the classification algorithm, the weight of the algorithm will fluctuate in the process of convergence, and it is easy to converge to the local optimal result. Therefore, in order to avoid this situation, $M F_{n}^{i}$ is normalized as follows:

$$
M F_{n}^{i^{\prime}}=\frac{\mathrm{MF}_{n}^{i}-\operatorname{mean}\left(\mathrm{MF}_{n}^{i}\right)}{\max \left(\mathrm{MF}_{n}^{i}\right)-\min \left(\mathrm{MF}_{n}^{i}\right)},
$$

where mean is the function of taking the mean value; $\mathrm{MF}_{n}^{i}$ is the multifeature vector extracted from the i-th sample of the $\mathrm{n}$-th subject; $\mathrm{MF}_{n}^{i^{\prime}}$ is the normalized result; $\mathrm{MF}_{n}^{i^{\prime}} \in[-1,1]$, which will greatly improve the convergence ability of the algorithm.

2.3. Matching. In this paper, the matching uses the following classification and Euclidean distance to produce their decisions.

2.3.1. Classification. Classification is a fundamental task in pattern recognition that involves the formation of a classifier. In this study, we use two commonly applied classifiers (NBC and LDC) to identify the class labels for subjects.

Naive Bayes [38] uses the well-known Bayes theorem to build a probabilistic model of the subject's features. The intuitive idea of an NBC is that future observations of a feature vector belonging to a subject will follow the same probabilistic distribution of feature vectors that were given for training for the same subject and that the value of a feature is independent of the value taken by other features. The basic idea of a linear discriminant classifier is to minimize the difference between the same class samples and maximize the difference between the different class samples by linear projection. In other words, after projection, the same class samples are gathered, while the different class samples are as far away as possible.

Here, the multifeature $\left(\left[\mathrm{MF}_{1}, \mathrm{MF}_{2}, \ldots, \mathrm{MF}_{M}\right], M\right.$ is the number of subjects) normalized is divided into training set 
and testing set, and then the two sets are directly input into the classifier, and the recognition rate is finally output.

2.3.2. Euclidean Distance. In this study, Euclidean distance is used to compute the similarity between two PPG features extracted, and its decision is produced. In other words, in this section, we describe the matching procedure for the testing data. In general, the testing data are divided into two separate sets: one is an enroll set, and the other is a probe set. The enroll set is regarded as the matching template, and the probe set is regarded as the query sample [39].

First, as illustrated in Section 2.1, the enroll and probe sets should be rerepresented. Then, multifeatures of the two sets are extracted, respectively, and the multifeatures normalized are denoted as $\mathrm{MF}_{\text {enroll }}$ and $\mathrm{MF}_{\text {probe }}$. Finally, for each probe, the Euclidean distance, which estimates the similarity between the probe and the enroll sample, is defined by the following formulation:

$$
d_{\text {euclidean }}(i, \mathrm{j})=\sqrt{\sum_{k=0}^{N-1}\left(\mathrm{MF}_{\text {probe }}^{i}(k)-\mathrm{MF}_{\text {enroll }}^{j}(k)\right)^{2}},
$$

where $\mathrm{MF}_{\text {probe }}^{i}(k)$ is a multifeature point of the i-th probe sample, $\mathrm{MF}_{\text {enroll }}^{j}(k)$ is a multifeature point of the $\mathrm{j}$-th enroll sample, $\mathrm{d}_{\text {euclidean }}(i, \mathrm{j})$ is the distance between the $\mathrm{i}$-th probe sample and the $\mathrm{j}$-th enroll sample, and $\mathrm{N}$ is the dimension of one multifeature vector. After $\mathrm{d}_{\text {euclidean }}(i, \mathrm{j})$ is acquired, we can determine whether the probe is an impostor or genuine by comparing $\mathrm{d}_{\text {euclidean }}(i, j)$ to a threshold. Varying the threshold adjusts the false acceptance rate (FAR) and false rejection rate (FRR), generating what is called a receiver operating characteristic (ROC) curve. The equal error rate (EER) is the point in the ROC curve at which the FAR is equal to the FRR. In general terms, the lower the EER, the better acceptance and protection against circumvention.

\section{Experiments and Results}

In the section, all experiments were conducted on a personal computer with Intel $^{\circledR}$ Core $^{\mathrm{TM}}$ i7 processor, $2.7 \mathrm{GHz}$ speed, and 8 GB RAM.

3.1. Data Acquisition. Performance of the proposed method was tested on the three PPG datasets including Beth Israel Deaconess Medical Center (BIDMC) [40,41], Multiparameter Intelligent Monitoring for Intensive Care (MIMIC) $[28,42]$, and CapnoBase $[43,44]$.

The BIDMC dataset comprises $538 \mathrm{~min}$ recordings of ECG, PPG, and so on, signals (sampling frequency, fs $=125 \mathrm{~Hz}$ ) acquired from adult patients (aged 19-90+, 32 females). The patients were randomly selected from a larger cohort that was admitted to medical and surgical intensive care units at the BIDMC, Boston, Mass., USA. The MIMIC database collects recordings of PLETH, ABP, RESP, and so on of patients in ICUs and is published on PhysioBank ATM for free. PLETH is the PPG data signal needed and its frequency is $125 \mathrm{~Hz}$. The partial recordings of 32 patients of
10 min duration downloaded by [28] are used for this work. The CapnoBase dataset contains PPG, ECG, and other signals for 42 cases of $8 \mathrm{~min}$ duration, and its sampling frequency is $300 \mathrm{~Hz}$.

3.2. Performance Evaluation Metrics. To evaluate the performance of the proposed method, we conducted experiments on two methods. For the identification problem, the recognition rate is used as the evaluation criterion, which is the percentage of correctly classified testing samples, defined as follows:

$$
\text { Recognition rate }=\frac{N_{\text {correct }}}{N_{\text {testing }}},
$$

where $\mathrm{N}_{\text {testing }}$ is the total number of testing samples and $\mathrm{N}_{\text {correct }}$ is the number of testing samples that are correctly classified. For the verification problem, the EER is the measure. EER is acquired from the FAR and FRR, which have the following definition:

$$
\begin{aligned}
& \mathrm{FAR}=\frac{\mathrm{FN}}{\mathrm{TN}+\mathrm{FN}}, \\
& \mathrm{FRR}=\frac{\mathrm{FP}}{\mathrm{TP}+\mathrm{FP}},
\end{aligned}
$$

where FAR is the false accept rate, FRR is the false reject rate, $\mathrm{FN}$ is false negative, TN is true negative, FP is false positive, and TP is true positive. EER is defined by the value where FAR is equal to FRR.

3.3. $W_{\text {size }}$ and $S_{\text {step }}$ Analysis. Since the size of sliding window $\left(W_{\text {size }}\right)$ and the step size of sliding $\left(S_{\text {step }}\right)$ have an important influence on the recognition rate, for choosing suitable the $W_{\text {size }}$ and $S_{\text {step }}$, two experiments based on the multifeature were performed on the BIDMC, MIMIC, and CapnoBase datasets. One experiment was sliding window size $\left(W_{\text {size }}\right)$ influence on the recognition rate. First, we set $S_{\text {step }}=1$, rerepresented the raw PPG data by changing $W_{\text {size }}$, and produced experimental samples of 10 groups. Then, $80 \%$ of the samples of each subject in each group were taken as a training set, and $20 \%$ of the samples were taken as a testing set. Finally, the two sets were directly sent to the classifiers including $\mathrm{k}-\mathrm{NN}, \mathrm{RF}, \mathrm{LDC}$, and $\mathrm{NBC}$ and output recognition rates. Figure 4 shows that, with the increase of $W_{\text {size }}$, the recognition rate is improved until it is stable. Another experiment was to detect the influence of $S_{\text {step }}$ on recognition rate. First, we randomly selected the sample points of 1.5 cycles as $W_{\text {size }}$, rerepresented the raw PPG data by the sliding window method according to $S_{\text {step }}=1,2,3, \ldots, 20$, respectively, and produced experimental samples of 20 groups. Then, $80 \%$ of the samples of each subject in each group were used as a training set, and $20 \%$ of the samples were used as a testing set. Finally, the two sets were directly sent to the classifiers including $\mathrm{k}-\mathrm{NN}, \mathrm{RF}, \mathrm{LDC}$, and NBC and output recognition rates. Figure 5 shows that, with the increase of $S_{\text {step }}$, the recognition rate decreases on the BIDMC dataset; 


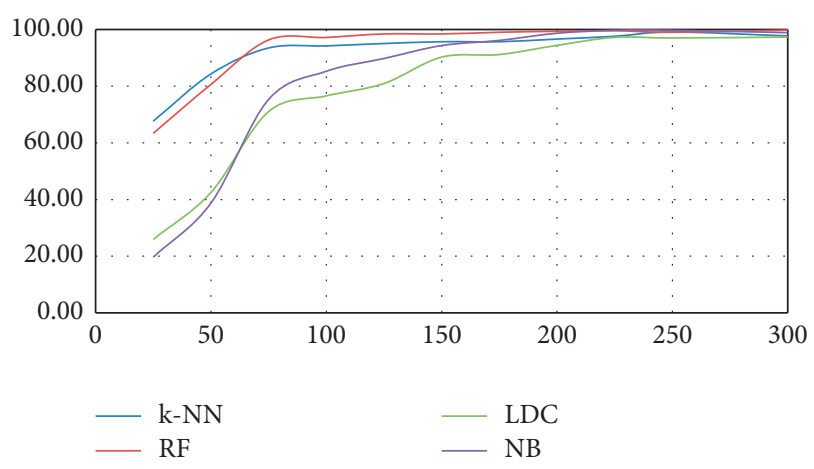

(a)

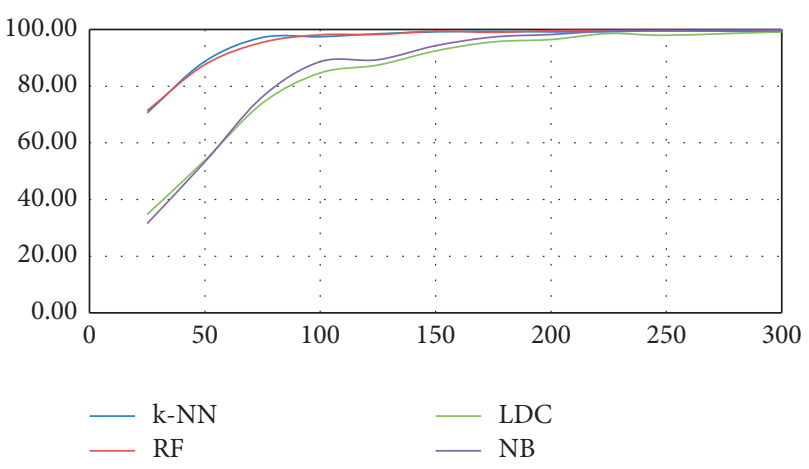

(b)

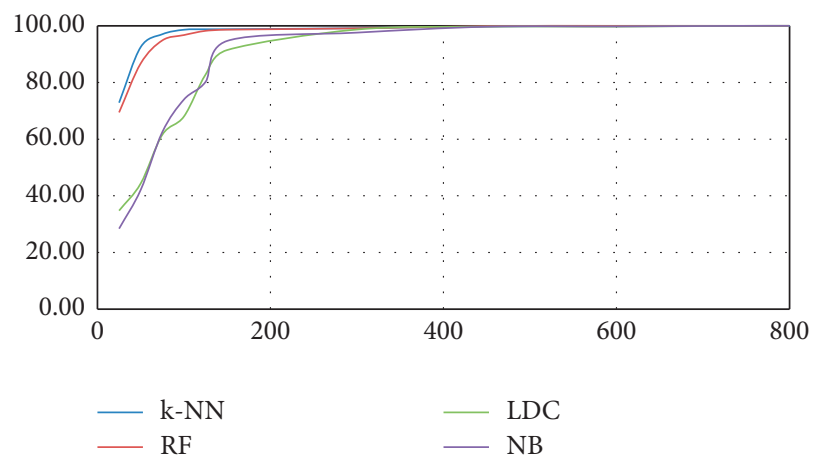

(c)

FIGURE 4: Influence of the $\mathrm{W}_{\text {size }}$ on recognition rate on the three datasets, (a) BIDMC, (b) MIMIC, and (c) CapnoBase. The horizontal axis shows the size of the sliding window, and the vertical axis represents the recognition rate.

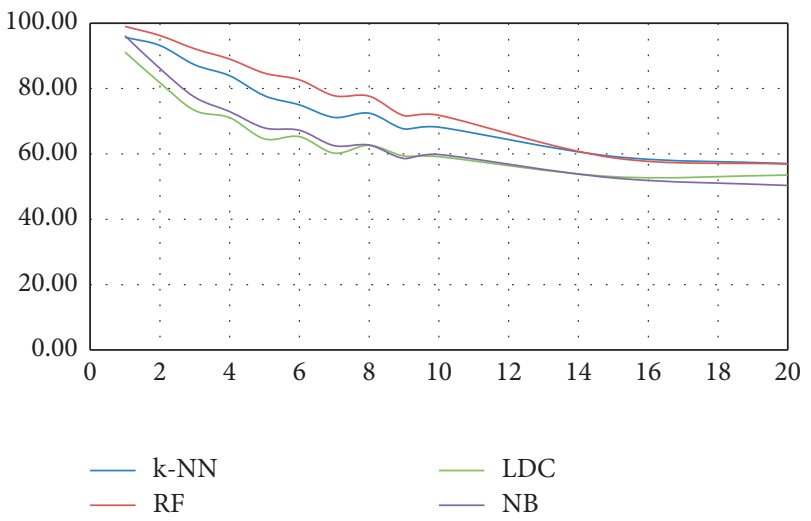

FIGURE 5: Influence of $S_{\text {step }}$ on recognition accuracy on the BIDMC dataset. The horizontal axis shows the size of the sliding step, and the vertical axis represents the recognition rate.

experiments show that they are the same on the MIMIC and CapnoBase.

As can be seen from Figure 4, when the sliding window size is chosen with the sample points of 1.5 cycles, the proposed method can achieve a relatively high and stable recognition rate on the classifiers including k-NN, RF, LDC, and NBC. In Figure 4(c), when the recognition rate is relatively stable, the number of sample points is relatively large, which should be caused by the high sampling frequency of the CapnoBase dataset. Figure 5 shows that the sliding step size is smaller and better. Therefore, in the following experiments, we choose the sample points of 1.5 cycles as $\mathrm{W}_{\text {size }}$, and $S_{\text {step }}=1$.

3.4. Performance of the Proposed Method. Because the testing data are directly sent to the classification method provided by MATLAB, it is not easy to operate the internal details of the classification method and report EER. Therefore, in this section, we not only did experiments based on classifiers but also did experiments based on Euclidean distance.

3.4.1. Experiments Based on Classifiers. MATLAB (R2018b) was used as the experimental programming environment. To avoid special cases, each experiment was run multiple times, and their average value was finally reported.

To evaluate the effectiveness of the features from various feature extraction, this experiment uses the LDC and NBC. Firstly, we used the method proposed in Section 2.1 to preprocess the raw PPG data. Then, the preprocessed data were divided into a training set ( $80 \%$ of the total data) and a testing set (20\%), and various features were extracted on the training set and testing set, respectively. Finally, the training set and the testing set were directly sent to the LDC and $\mathrm{NBC}$, and the recognition rates were output. Table 4 shows the experimental results on the three datasets.

Table 4 shows that the method proposed for extracting TDF is effective, and the features are distinguishable, so it has a high recognition rate on the LDC and NBC. The methods of frequency-domain and wavelet feature 
TABLE 4: Recognition rates of the various features combined with LDC and NBC on the three datasets.

\begin{tabular}{lccc}
\hline \multirow{3}{*}{ Dataset } & & \multicolumn{2}{c}{ Recognition rate } \\
& Feature vector & LDC & NBC \\
\hline \multirow{4}{*}{ BIDMC } & TDF & 87.33 & 96.73 \\
& FDF & 33.24 & 27.23 \\
& WF & 43.15 & 40.63 \\
& TDF and FDF & 89.37 & 97.48 \\
TDF and WF & 92.86 & 98.33 \\
FDF and WF & 64.84 & 55.13 \\
MF & 93.90 & 98.65 \\
TDF & 89.38 & 93.85 \\
MIMIC & FDF & 38.23 & 28.18 \\
& WF & 57.82 & 65.94 \\
TDF and FDF & 92.24 & 94.17 \\
TDF and WF & 94.32 & 97.40 \\
FDF and WF & 70.94 & 74.27 \\
MF & 95.42 & 97.76 \\
TDF & 99.04 & 99.12 \\
FDF & 43.25 & 37.86 \\
WF & 70.48 & 78.75 \\
CapnoBase & TDF and FDF & 99.12 & 99.21 \\
& TDF and WF & 99.26 & 99.34 \\
FDF and WF & 79.53 & 82.98 \\
MF & 99.47 & 99.69 \\
\hline
\end{tabular}

extraction are not ideal, but wavelet feature or FDF cannot be ignored in PPG biometrics, since they have better recognition performance when they are combined with other features (such as TDF). The results also show that the performance of combinational features is better than that of a single feature, especially the multifeature extracted that has good performance in PPG biometrics. On the three datasets, NBC is better than LDC with the same features extracted. It should be noted that the recognition rate of single wavelet feature on LDC and NBC is not high, which may be due to the overlapping of useful signal and noise frequency bands in the process of raw data preprocessing.

3.4.2. Experiments Based on Euclidean Distance. To account the usability-security trade-off, we conducted this experiment in Python 3.6 and reported the ROC and EER curves and so on. Firstly, we used the proposed method in Section 2.1 to preprocess the raw PPG data. Secondly, 10 subjects were randomly selected from the preprocessed data, and 10 samples of each of which were randomly selected. Thirdly, various features were extracted from all samples of the selected subjects and divided into an enroll set and a probe set. Fourthly, the Euclidean distance between the enroll set and the probe set is calculated by formula (7). Finally, the threshold is varied to calculate EER and recognition rate. Figures 6 and 7 show the ROC curve and EER curve of randomly selected testing data on the three datasets, respectively. The ROC curve represents the trade-off between FAR and FRR, while EER is generally adopted as a unique measure for characterizing the performance level of a biometrics system. The EER can be seen in the figure where the FAR and FRR cross each other. Table 5 shows the

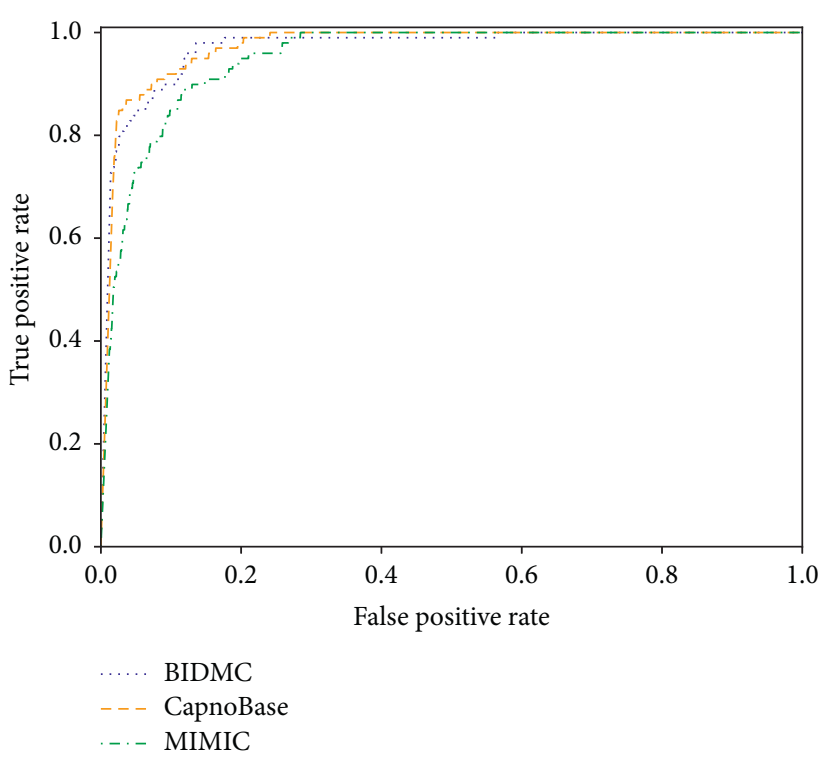

FIgURE 6: ROC curve.

recognition rate and EER of the proposed method on three datasets.

These experimental results further show that the multifeature extracted has high discrimination, and the recognition rate and EER based on the multifeature are the best on the three datasets. As shown in Table 5, in the PPG biometrics, the performance based on TDF and FDF and TDF and WF is superior to that of TDF, that based on TDF and WF is slightly superior to TDF and FDF, and that based on MF is optimal, which confirms that extracting the multifeature is very effective in PPG representation. The results of this experiment are consistent with those based on the LDC and NBC, which also demonstrates the effectiveness of the proposed method.

3.4.3. Friedman Test of the Multifeature. Friedman test $[45,46]$ is a two-way variance analysis, which can be used to test whether multiple related samples come from the same subject. In this section, we use the Friedman test to analyse the significance of the multifeatures extracted. For this reason, we did two experiments on the Friedman test. One was about intersubject; firstly, we randomly chose 10 subjects from a dataset and then extracted multifeature for each subject by our method to produce 10 samples; thirdly, the 10 samples were tested with the "K related samples..." of IBM SPSS Statistics; finally, the significance was reported. The other was about intrasubject; firstly, we randomly chose one subject from a dataset and then randomly chose 10 samples from the extracted multifeature samples of the subject; thirdly, the 10 samples were tested with the " $K$ related samples..." of IBM SPSS Statistics; finally, the significance was reported. Table 6 reports the test results of the two experiments on three datasets including BIDMC, MIMIC, and CapnoBase.

Generally, when the significance is less than 0.05 or 0.01 , the difference among samples is considered to be significant 


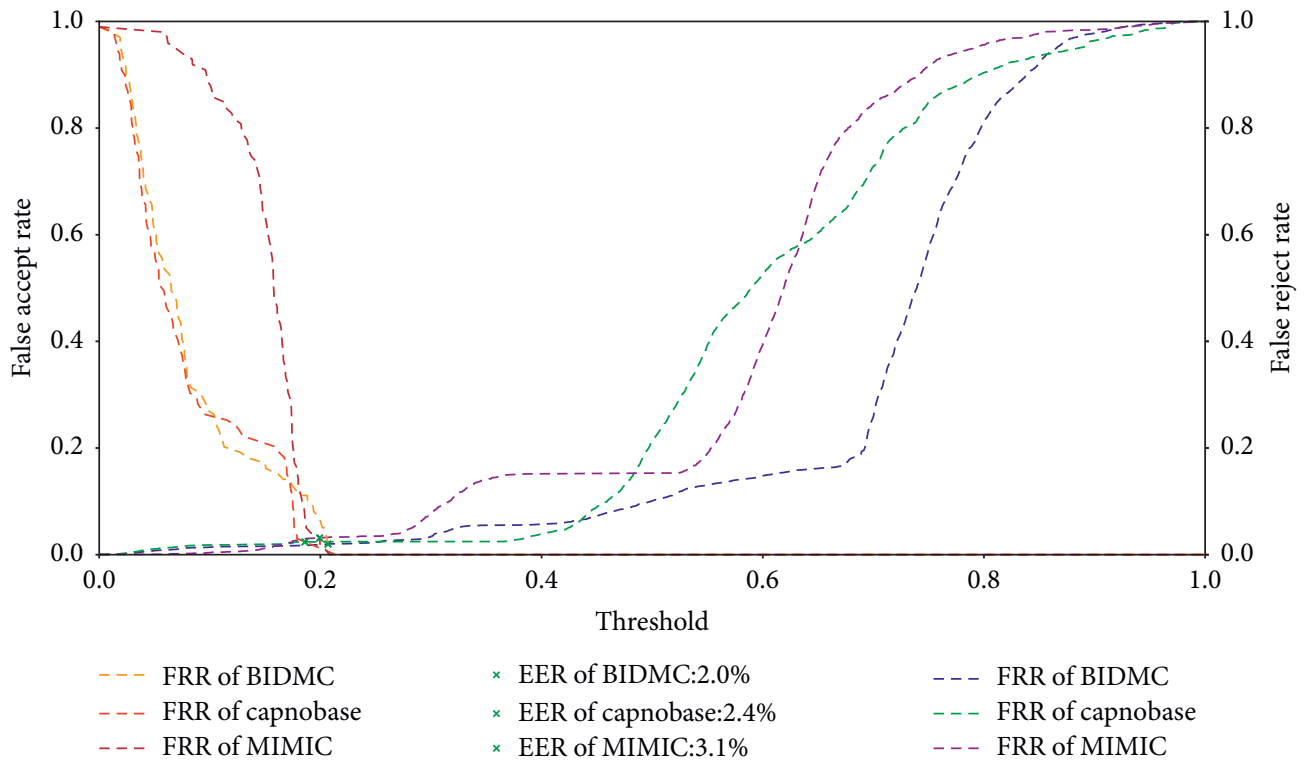

FIGURE 7: EER curve.

TABLE 5: Recognition rates and EERs based on the various features and Euclidean distance on the three datasets.

\begin{tabular}{lccc}
\hline Dataset & Feature vector & Recognition rate (\%) & EER (\%) \\
\hline \multirow{4}{*}{ BIDMC } & TDF & 96.08 & 3.52 \\
& TDF and FDF & 96.47 & 3.48 \\
& TDF and WF & 96.71 & 3.29 \\
& MF & 98.06 & 1.94 \\
MIMIC & TDF & 96.67 & 3.43 \\
& TDF and FDF & 96.86 & 3.18 \\
& TDF and WF & 96.87 & 3.14 \\
& MF & 96.99 & 3.11 \\
CapnoBase & TDF & 97.59 & 2.43 \\
& TDF and FDF & 97.59 & 2.41 \\
& TD and WF & 97.60 & 2.39 \\
& MF & 97.62 & 2.36 \\
\hline
\end{tabular}

Table 6: Significances of the intersubject and intrasubject on the three datasets.

\begin{tabular}{lcc}
\hline Dataset & \multicolumn{2}{c}{ Significance } \\
& Intersubject & Intrasubject \\
\hline BIDMC & 0.000 & 0.291 \\
MIMIC & 0.000 & 0.054 \\
CapnoBase & 0.000 & 0.147 \\
\hline
\end{tabular}

or extremely significant; when the significance is greater than 0.05 , it is considered to be not significant. As shown in Table 6, on the three datasets, the difference of intersubject is extremely significant, while the difference of intrasubject is not significant; this conclusion is highly consistent with our other experiments.
3.5. Comparisons with the State-of-the-Art Methods. We perform comparisons between our method and the state-ofthe-art methods on the three datasets. Karimian et al. [18] used a nonfiducial approach for PPG with a DWT and k-NN and reported $99.84 \%$ recognition rate with an EER of $1.31 \%$ on the CapnoBase dataset. Jorge et al. [22] studied several feature extractors (e.g., cycle average, multicycle based on the time-domain, and the Karhunen-Loève transform average) and matching metrics (Manhattan and Euclidean distances) that had been tested by using the CapnoBase, and an optimal EER of 1.0\% was achieved. Lee et al. [30] tried to use a DCT for extracting features from the preprocessed PPG data, and the extracted features were used as the input variables for RF; the recognition rate was $99 \%$ on the CapnoBase. Yang et al. [28] extracted the three-layer feature based on the SSV on three datasets, and the features were used as the input variables for NBC and achieved recognition rates of $98.66 \%, 97.15 \%$, and $99.71 \%$ on the BIDMC, MIMIC, and CapnoBase, respectively. The experimental results of $[18,22,28,30]$ are directly reported from previous original work and are summarized in Table 7.

As shown in Table 7, although some of our methods are not the best, they have achieved a high recognition rate on the three datasets. Compared with [18], the recognition rate of their method is slightly superior, which may be that the two-step feature selection based on Kolmogorov Smirnov (KS) and kernel principal component analysis (KPCA) used in the feature-extraction module [18] is more effective. However, our method only makes a simple combination of time-domain, frequency-domain, and wavelet features, so that the multifeature is not enough distinguishable, which is what we will improve in the future. Compared with [28], 
TABLE 7: Comparison of the proposed method and state-of-the-art methods on the three datasets.

\begin{tabular}{|c|c|c|c|c|}
\hline Dataset & Method & Matching & Recognition rate $(\%)$ & EER (\%) \\
\hline \multirow{2}{*}{ BIDMC } & {$[28]$} & NBC & 98.66 & - \\
\hline & Proposed & NBC & 98.65 & - \\
\hline \multirow{2}{*}{ MIMIC } & {$[28]$} & NBC & 97.15 & - \\
\hline & Proposed & NBC & 97.76 & - \\
\hline \multirow{5}{*}{ CapnoBase } & {$[18]$} & $\mathrm{k}-\mathrm{NN}$ & 99.84 & 1.31 \\
\hline & {$[30]$} & RF & 99.00 & - \\
\hline & {$[22]$} & Manhattan and Euclidean distance & - & 1.00 \\
\hline & {$[28]$} & NBC & 99.71 & - \\
\hline & Proposed & $\mathrm{NBC}$ & 99.69 & - \\
\hline
\end{tabular}

TABLE 8: Comparison of the proposed method and [28] method in time consumption.

\begin{tabular}{lcc}
\hline Method & Process & Time $(\mathrm{s})$ \\
\hline \multirow{3}{*}{ Proposed } & Preprocessing & 0.000116 \\
& Feature extraction & 0.026431 \\
& Matching & 0.000264 \\
{$[28]$} & Preprocessing & 0.000107 \\
& Feature extraction & 0.451316 \\
& Matching & 0.001217 \\
\hline
\end{tabular}

although the recognition rate of their method is slightly higher, they obtain the better result at the cost of more feature-extraction time, which is mainly due to [28] using the deep cascade model $[47,48]$ in the process of three-layer feature extraction. However, our method is mainly some simple mathematical calculation; the time cost is very lower.

3.6. Time-Cost Analysis. To verify the efficiency of the proposed framework, the preprocessing time (in seconds), feature-extraction time, and matching time cost during the PPG biometrics procedure on the MIMIC dataset are further summarized. The results are summarized in Table 8 . The experimental environment includes HP EliteBook 8570w Notebook with Intel ${ }^{\circledR}$ Core $^{\mathrm{TM}}$ i7 processor, $2.7 \mathrm{GHz}$ speed, 8 GB RAM, the 64-bit Windows 7 operating system, and MATLAB (2018b). Since the source codes of the other methods except [28] are not available for us, Table 8 only lists the average preprocessing time of one sample, average feature-extraction time of one sample, average matching time of one sample pair in the method of [28], and ours. From the experiments, the preprocessing time and the matching time are almost the same using the two methods; compared to the method of [28], the feature-extraction time of the proposed method is much less than theirs, which is clipped more than 0.4 seconds. It can be obvious that our method is more ideal for feature extraction.

\section{Conclusions and Future Work}

In this study, we propose a novel PPG biometrics framework. Firstly, we use the sliding window method to rerepresent the PPG raw data to avoid losing useful information due to denoising and analyse the influence of sliding window size and sliding step size on the recognition rate. Secondly, the time-domain, frequency-domain, and wavelet features are extracted and combined to form the multifeature, which is to make up for the lack of a single feature extracted. Finally, the normalized multifeature is matched by LDC, NBC, and Euclidean distance, and it is to make a decision. The extensive experimental results demonstrate that the method based on multifeature and NBC can achieve high recognition rates of $98.65 \%, 97.76 \%$, and $99.69 \%$ on the BIDMC, MIMIC, and CapnoBase datasets, respectively, and it can be seen that our method is not inferior to several stateof-the-art methods. It should be noted that the multifeature can also achieve a high recognition rate using LDC on the three datasets. The extensive experimental results on the three datasets also demonstrate that the PPG biometrics based on the multifeature and Euclidean distance can provide a lower EER and high recognition rate. In particular, our method is suitable for small-scale PPG data biometrics and consumes fewer resources.

Despite the satisfactory performance achieved by our method, there is still some room for the proposed PPG biometrics framework. For example, the cost time of multifeature extraction needs to be improved, especially wavelet feature-extraction time, which is above $95 \%$ of the multifeature-extraction time; the fusion method of time-domain, frequency-domain, and wavelet features needs to be improved; how to apply PPG biometrics with large-scale data. In addition, our method can be used for reference for other biometrics or for more in-depth biometrics research. In our future work, we will further explore the attributive information of the PPG signal to improve the performance [49].

\section{Data Availability}

The simulated data used to support the simulation part of this study are available from the corresponding author upon request, and the real-world PPG data can be obtained from https://www.physionet.org/physiobank/database/bidmc, https://archive.physionet.org/cgi-bin/ATM? database $=$ mimic $2 \mathrm{db}$, and https://dataverse.scholarsportal. info/dataverse/capnobase.

\section{Conflicts of Interest}

The authors declare no conflicts of interest. 


\section{Acknowledgments}

This work was supported in part by the NSFC-Xinjiang Joint Fund under Grant U1903127 and in part by the Key Research and Development Project of Shandong Province under Grant 2018GGX101032.

\section{References}

[1] C. Tao and G. Liu, "An efficient fingerprint preprocessing algorithm based on FDCT," Journal of Computational Information Systems, vol. 6, no. 12, pp. 4055-4063, 2010.

[2] R. Brunelli and T. Poggio, "Face recognition: features versus templates," IEEE Transactions on Pattern Analysis and Machine Intelligence, vol. 15, no. 10, pp. 1042-1052, 1993.

[3] D. Dumn, "Using a multi-layer perceptron neural for human voice identification," ", in Proceedings of the 4th International Conference on Signal Processing, Applications and Technology, Newton, MA, USA, 1993.

[4] M. Negin, T. A. Chmielewski, M. Salganicoff, U. M. Von Seelen, P. L. Venetainer, and G. G. Zhang, "An iris biometric system for public and personal use," Computer, vol. 33, no. 2, pp. 70-75, 2000.

[5] K. S. Paik, C. H. Chung, J. O. Kim, and D. J. Hwang, "On a lip print recognition by the pattern kernels with multiresolution architecture," Proceedings of International Conference on Image Processing, vol. 2, pp. 246-249, 2001.

[6] C. Y. Yam, M. S. Nixon, and J. N. Carter, "Performance analysis on new biometric gait motion model," in Proceedings of the 5th IEEE Southwest Symposium on Image Analysis and Interpretation, pp. 31-34, Santa Fe, New Mexico, 2002.

[7] L. Mirmohamadsadeghi and A. Drygajlo, "Palm vein recognition with local texture patterns," IET Biometrics, vol. 3, no. 4, pp. 198-206, 2014.

[8] L. Yang, G. Yang, X. Xi, K. Su, Q. Chen, and Y. Yin, "Finger vein code: from indexing to matching," IEEE Transactions on Information Forensics and Security, vol. 14, no. 5, pp. 12101223, 2019.

[9] R. B. Paranjape, J. Mahovsky, L. Benedicenti, and Z. Koles, "The electroencephalogram as a biometric," Canadian Conference on Electrical and Computer Engineering, vol. 2, pp. 1363-1366, 2001.

[10] L. Biel, O. Pettersson, L. Philipson, and P. Wide, "ECG analysis: a new approach in human identification," IEEE Transactions on Instrumentation and Measurement, vol. 50, no. 3, pp. 808-812, 2001.

[11] Y. Huang, G. Yang, K. Wang, H. Liu, and Y. Yin, "Learning Joint and specific patterns: a unified sparse representation for off-the-person ECG biometric recognition," IEEE Transactions on Information Forensics and Security, vol. 16, pp. 147-160, 2021.

[12] N. Akhter, S. Tharewal, V. Kale, A. Bhalerao, and K. V. Kale, "Heart-based biometrics and possible use of heart rate variability in biometric recognition systems," in Advanced Computing and Systems for Security, R. Chaki, A. Cortesi, K. Saeed, and N. Chaki, Eds., Vol. vol 395, Springer, New Delhi, India, 2016.

[13] A. Bonissi, R. D. Labati, L. Perico, R. Sassi, F. Scotti, and L. Sparagino, "A preliminary study on continuous authentication methods for photoplethysmographic biometrics," in Proceedings of the 2013 IEEE Workshop on Biometric Measurements and Systems for Security and Medical Applications (BIOMS), pp. 28-33, Napoli, Italy, September 2013.
[14] A. R. Kavsaoğlu, K. Polat, and M. R. Bozkurt, “A novel feature ranking algorithm for biometric recognition with PPG signals," Computers in Biology and Medicine, vol. 49, pp. 1-14, 2014.

[15] M. Elgendi, "On the analysis of fingertip photoplethysmogram signals," Current Cardiology Reviews, vol. 8, no. 1, pp. 14-25, Jun. 2012.

[16] D. Y. Hwang, B. Taha, D. S. Lee et al., "Evaluation of the time stability and uniqueness in PPG-based biometric system," IEEE Transactions on Information Forensics and Security, vol. 99, p. 1, 2020.

[17] J. Yao, X. Sun, and Y. Wan, "A pilot study on using derivatives of photoplethysmographic signals as a biometric identifier," in Proceedings of the 29th IEEE Annual Int. Conf. of the Engineering in Medicine and Biology Society (EMBS), pp. 45764579, Québec, Canada, August 2007.

[18] N. Karimian, M. Tehranipoor, and D. Forte, "Non-fiducial PPG-based authentication for healthcare application," in Proceedings of the 2017 IEEE EMBS International Conference on Biomedical Health Informatics (BHI), pp. 429-432, Orland, FL, USA, February 2017.

[19] N. I. Nadzri, K. A. Sidek, and A. F. Ismai, "Biometric recognition for twins inconsideration of age variability using PPG signals," Journal of Telecommunication, Electronic and Computer Engineering, vol. 10, pp. 97-100, 2018.

[20] G. Lovisotto, H. Turner, S. Eberz, and I. Martinovic, "Seeing red: PPG biometrics using smartphone cameras," in Proceedings of the IEEE/CVF Conference on Computer Vision and Pattern Recognition (CVPR) Workshops, pp. 818-819, Washington, DC, USA, 2020.

[21] U. Yadav, S. N. Abbas, and D. Hatzinakos, "Evaluation of PPG biometrics for authentication in different states," in Proceedings of the EEE 2018 International Conference on Biometrics (ICB), pp. 277-282, Gold Coast, Australia, February 2018.

[22] J. Sancho, Á. Alesanco, and J. García, "Biometric authentication using the PPG: a long-term feasibility study," Sensors, vol. 18, no. 5, pp. 1525-13, 2018.

[23] A. Al-Sidani, B. Ibrahim, A. Cherry et al., "Biometric identification using photoplethysmography signal," in Proceedings of the the Third International Conference on Electrical and Biomedical Engineering, Clean Energy and Green Computing (EBECEGC2018), pp. 12-15, Beirut, Lebanon, April 2018.

[24] N. A. L. Jaafar, K. A. Sidek, and S. N. A. M. Azam, "Acceleration plethysmogram based biometric identification," in Proceedings of the 2015 Int. Conf. on BioSignal Analysis, Processing and Systems (ICBAPS), pp. 26-28, Kuala Lumpur, Malaysia, May 2015.

[25] S. Chakraborty and S. Pal, "Photoplethysmogram signal based biometric recognition using linear discriminant classifier," in Proceedings of the IEEE 2nd International Conference on Control, Instrumentation, Energy \& Communication (CIEC), pp. 183-187, Kolkata, India, January 2016.

[26] X. Zhang, Z. Qin, and Y. Lyu, "Biometric authentication via finger photoplethysmogram," in Proceedings of the 2018 2nd International Conference 2018, Beirut, Lebanon, April 2018.

[27] P. Spachos, J. Gao, and D. Hatzinakos, "Feasibility study of photoplethysmographic signals for biometric identification," in Proceedings of theIEEE 2011 17th International Conference on Digital Signal Processing (DSP), pp. 1-5, Corfu, Greece, July 2011.

[28] J. Yang, Y. Huang, F. Huang, and G. Yang, "Photoplethysmography biometric recognition model based on sparse softmax vector and k-nearest neighbor," Journal of 
Electrical and Computer Engineering, vol. 2020, Article ID 9653470, 9 pages, 2020.

[29] P. Farago, R. Groza, L. Ivanciu et al., "A correlation-based biometric identification technique for ECG, PPG and EMG," in Proceedings of the 2019 15th International Conference on Telecommunications (ConTEL), pp. 716-719, Graz, Austria, July 2019.

[30] S.-W. Lee, D. K. Woo, Y. K. Son et al., "Wearable bio-signal(PPG)-Based personal authentication method using random forest and period setting considering the feature of PPG signals," Journal of Computers, vol. 14, no. 4, pp. 283-294, 2019.

[31] V. Jindal, J. Birjandtalab, M. B. Pouyan et al., "An adaptive deep learning approach for PPG-based identification," in Proceedings of the 2016 38th Annual International Conference of the IEEE Engineering in Medicine and Biology Society (EMBC'16), pp. 6401-6404, Orlando, Florida, USA, August 2016.

[32] L. Everson, D. Biswas, M. Panwar et al., "Biometricnet: deep learning based biometric identification using wrist-worn ppg," in Proceedings of the 2018 IEEE International Symposium on Circuits and Systems (ISCAS), pp. 1-5, Florence, Italy, May 2018.

[33] D. Biswas, L. Everson, M. Liu et al., "Deep learning framework for PPG-based heart rate estimation and biometric identification in ambulant environmen," Ieee Transactions On Biomedical Circuits And Systems, vol. 2, 2019.

[34] D. Y. Hwang and D. Hatzinakos, "PPSNet: PPG-based personalized verification system," in Proceedings of the 2019 IEEE Canadian Conference of Electrical and Computer Engineering (CCECE), pp. 1-4, Edmonton, Canada, May 2019.

[35] El-Badawy, M. Ismail, O. P. Singh et al., "Automatic classification of regular and irregular capnogram segments using time- and frequency-domain features: a machine learningbased approach," Technology and Health Care, vol. 29, no. 2, 2020.

[36] K. G. Kharate and V. H. Patil, "Color image compression based on wavelet packet best tree," International Journal of Computer Ence Issuesabs, vol. 1004, p. 3276, 2010.

[37] https://www.mathworks.com/help/wavelet/ug/waveletpackets.html.

[38] J. Blasco, T. M. Chen, J. Tapiador, and P. Peris-Lopez, "A survey of wearable biometric recognition systems," ACM Computing Surveys (CSUR), vol. 49, no. 3, p. 43, 2016.

[39] K. K. Wang, G. P. Yang, Y. W. Huang, and Y. L. Yin, "Multiscale differential feature for ECG biometrics with collective matrix factorization," Pattern Recognition, vol. 102, 2020.

[40] M. A. F. Pimentel, A. E. W. Johnson, P. H. Charlton et al., "Toward a robust estimation of respiratory rate from pulse oximeters," IEEE Transactions on Biomedical Engineering, vol. 64, no. 8, pp. 1914-1923, 2017.

[41] A. L. Goldberger, L. Amaral, L. Glass et al., "Components of a new research resource for complex physiologic signals," Circulation, vol. 101, no. 23, pp. 215-220, 2000.

[42] https://archive.physionet.org/cgi-bin/ATM?database=\% 20 mimic $2 \mathrm{db}$.

[43] http://www.capnobase.org/database/pulse-oximeter-ieeetbme-benchmark.

[44] W. Karlen, S. Raman, J. M. Ansermino, and G. A. Dumont, "Multiparameter respiratory rate estimation from the photoplethysmogram," IEEE Transactions on Biomedical Engineering, vol. 60, no. 7, pp. 1946-1953, 2013.

[45] M. Friedman, "The use of ranks to avoid the assumption of normality implicit in the analysis of variance," Journal of the
American Statistical Association, vol. 32, no. 200, pp. 675-701, 1937.

[46] S. Sakamoto, A. Lala, T. Oda, V. Kolici, L. Barolli, and F. Xhafa, "Analysis of WMN-HC simulation system data using friedman test," in Proceedings of the 2015 Ninth International Conference on Complex, Intelligent, and Software Intensive Systems, pp. 254-259, Santa Catarina, Brazil, 2015.

[47] J. Liu and L. Zhang, "Sparse softmax vector coding based deep cascade model," in Proceedings of the The Chinese Conference on Computer Vision (CCCV2017), pp. 603-614, Tianjin, China, October 2017.

[48] L. Zhang, J. Liu, B. Zhang et al., "Deep cascade model-based face recognition: when deep-layered learning meets small data," IEEE Transactions on Image Processing, vol. 29, pp. 1016-1029, 2019.

[49] Y. Y. Gu, Y. Zhang, and Y. T. Zhang, "A novel biometric approach in human verification by photoplethysmographic signals," in Proceedings of theInternational IEEE Embs Special Topic Conference on Information Technology Applications in Biomedicine, pp. 13-14, Prague, Czech Republic, 2003. 